Pacific Journal of Mathematics

COVERING THEOREMS FOR FINITE NONABELIAN SIMPLE 


\section{COVERING THEOREMS FOR FINITE NONABELIAN SIMPLE GROUPS. V.}

\section{J. L. Brenner, R. M. Cranwell, and J. Riddell}

In the alternating group $A_{n}, n=4 k+1>5$, the class $C$ of the cycle $(12 \cdots n)$ has the property that $C C$ covers the group. For $n=16 k$ there is a class $C$ of period $n / 4$ in $A_{n}$ such that $C C$ covers $A_{n} ; C$ is the class of type $(4 k)^{4}$.

1. Introduction. It was shown by E. Bertram [1] that for $n \geqq 5$ every permutation in $A_{n}$ is the product of two $l$-cycles, for any $l$ satisfying $[3 n / 4] \leqq l \leqq n$. Hence $A_{n}$ can be covered by products of two $n$-cycles and also by products of two $(n-1)$-cycles. But if $n$ is odd the $n$-cycles in $A_{n}$ fall into two conjugate classes $C, C^{\prime}$, and similarly for the $(n-1)$-cycles if $n$ is even, so that the quoted result does not decide whether

$$
C C=A_{n} .
$$

The question was decided affirmatively for $n=4 k+2$ and negatively for $n=4 k, 4 k-1$ in [2]. The question is now decided affirmatively in the remaining case $n=4 k+1, n \neq 5$.

THEOREM 1. For $n=4 k+1>5$, the class $C$ of the cycle $(12 \cdots n)$ has property (1).

The proof is in $\$ \$ 2-4$.

Regarding the product $C C^{\prime}$, it was shown in [2] that $C C^{\prime}$ covers $A_{n}(n \geqq 5)$ if $n=4 k, 4 k-1$, while if $n=4 k+1,4 k+2, C C^{\prime}$ contains all of $A_{n}$ but the identity.

By an argument quite similar to the proof of Theorem 1, we have proved

THEOREM 2. For $n=16 k$, the class $C$ of type $(4 k)^{4}$ in $A_{n}$ has property (1).

The proof and some related matters are discussed in $\S 5$. Note that the class in Theorem 2 has period $n / 4$.

2. The case $n=9$. Let $a=(123456789)$. For every class in $A_{9}$, a conjugate $b$ of $a$ can be found such that $a b$ represents (lies in) that class. This assertion is the substance of the table below. 


\begin{tabular}{ll}
\multicolumn{1}{c}{$b$} & \multicolumn{1}{c}{$a b$} \\
$\begin{array}{c}a^{-1} \\
(193248765)\end{array}$ & \multicolumn{1}{c}{$(14)(38)$} \\
$(176235894)$ & $(13)(25)(48)(79)$ \\
$(132987654)$ & $(193)$ \\
$(134765289)$ & $(18)(24)(379)$ \\
$(132798465)$ & $(174)(369)$ \\
$(184523796)$ & $(135)(274)(698)$ \\
$(137259486)$ & $(15)(276)(3849)$ \\
$(123794865)$ & $(1384)(2769)$ \\
$(132798654)$ & $(17693)$ \\
$(189623574)$ & $(13)(25)(47986)$ \\
$(132869745)$ & $(18764)(359)$ \\
$(132845697)$ & $(18746)(359)$ \\
$(159348726)$ & $(162495)(38)$ \\
$(186974532)$ & $(3598764)$ \\
$a$ & $(135792468) \sim a$ \\
$(125678934)$ & $(315792468)$
\end{tabular}

3. A lemma. In $\S 3$ and $\S 4, C$ will denote the class of the cycle $a=(12 \cdots n)$ in $A_{n}$.

Lemma. If $n=4 k+1>5$, then CC contains the type $2^{2 k} 1^{1}$.

Proof. If $n \equiv 1(\bmod 8)$, then $x=$

$$
(n n-3 n-2 n-1, n-4 n-7 n-6 n-5 ; \cdots ; 9678,5234 ; 1)
$$

is conjugate to $a$ and

$$
a x=(13)(24)(57)(68) \cdots(n-4 n-2)(n-3 n-1) .
$$

If $n \equiv 5(\bmod 8), n>13$, then $y=$

$$
(n n-3 n-2 n-1, n-4 n-7 n-6 n-5 ; \cdots ; 21181920 \text {, }
$$

$17141516 ; 139610,127811 ; 5234,1)$

is conjugate to $a$ and

$$
\begin{gathered}
a y=(13)(24)(510)(68)(711)(912)(1315)(1416) \cdots \\
(n-4 n-2)(n-3 n-1)
\end{gathered}
$$


If $n=13$ use the last 13 letters of the above $y$. (The pattern of $y$ differs from that of $x$ only in the last block of 8 letters between semi-colons, $139 \cdots 11$, in which the number of reversals is odd, whereas in every other such block of 8 letters in either $x$ or $y$, the number of reversals is even.)

4. The induction. The induction proceeds from $n-4$ to $n=4 k+1$. The induction hypothesis is: For every permutation $T$ in $A_{n-4}$, there are two $(n-4)$-cycles $d_{1}$ and $d_{2}$, both in the class of the $(n-4)$-cycle $(12 \cdots n-6 n-5 n-4)$, and also two other $(n-4)$ cycles $d_{1}^{\prime}$ and $d_{2}^{\prime}$, both in the class of $(12 \cdots n-6 n-4 n-5)$, such that $T=d_{1} d_{2}=d_{1}^{\prime} d_{2}^{\prime}$.

Let $S(\neq 1)$ be a permutation in $A_{n}$. To show that $C C$ contains $S$ we consider several cases. In each case we find a conjugate $S_{1}$ of $S$, and a certain permutation $g$ in $A_{n}$, such that $T=S_{1} g^{-1}$ fixes the letters $n, n-1, n-2, n-3$ and thus its restriction to $1,2, \cdots, n-4$ lies in $A_{n-4}$.

Case 1. $S$ contains a cycle with 5 or more letters: take

$$
g=(n n-1 n-2 n-3 n-4) \text {. }
$$

Case 2. $S$ contains no cycle with 5 or more letters, but $S$ contains at least one cycle with 4 letters: take

$$
g=(n n-1 n-2 n-3)(n-4 n-5) .
$$

Case 3. $S$ contains no cycle with more than 3 letters, but $S$ does contain two 3-cycles: take

$$
g=(n n-1 n-2)(n-3 n-4 n-5) .
$$

Case 4. $S$ is of type $3^{1} 2^{2 k-2} 1^{2}$ : take

$$
g=(n n-1 n-2)
$$

Now, if $S$ contains no cycle longer than a transposition, either $S$ is of type $2^{2 k} 1^{1}$, whence $C C$ contains $S$ by the lemma, or we have

Case 5. $S$ fixes 5 or more letters: take $g=1$.

The argument in Case 5 is quite simple. Since $S$ fixes 5 or more letters, $S$ has a conjugate $S_{1}$ that fixes $n, n-1, n-2, n-3$. Hence by the induction hypothesis $S_{1}=d_{1} d_{2}$, where $d_{1}$ and $d_{2}$ both fix $n, n-1, n-2, n-3$, and can be expressed 


$$
d_{1}=\left(a_{1} a_{2} \cdots a_{n-5} n-4\right), \quad d_{2}=\left(b_{1} b_{2} \cdots b_{n-5} n-4\right),
$$

where the permutation $a_{i} \rightarrow b_{i}$ is an even permutation of the letters $1,2, \cdots, n-5$. Then $S_{1}=d_{3} d_{4}$, with

$$
\begin{aligned}
& d_{3}=\left(a_{1} a_{2} \cdots a_{n-5} n n-1 n-2 n-3 n-4\right), \\
& d_{4}=\left(b_{1} b_{2} \cdots b_{n-5} n-4 n-3 n-2 n-1 n\right),
\end{aligned}
$$

and $d_{3}, d_{4}$ belong to the same class, be it $C$ or $C^{\prime}$. If the other part of the induction hypothesis is used in a similar fashion, the assertion that $C C$ contains $S$ follows.

The details for Case 1 are as follows. Since $T=S_{1} g^{-1}$ moves at most the first $n-4$ letters, we have by the induction hypothesis $T=d_{1} d_{2}=d_{1}^{\prime} d_{2}^{\prime}$ where $d_{1}, d_{2}\left[d_{1}^{\prime}, d_{2}^{\prime}\right]$ are from the same class in $A_{n-4}$. Writing

$$
d_{1}=\left(a_{1} a_{2} \cdots a_{n-5} n-4\right), \quad d_{2}=\left(b_{1} b_{2} \cdots b_{n-5} n-4\right),
$$

the permutation $a_{i} \rightarrow b_{i}$ is an even permutation of $1,2, \cdots, n-5$. Now $S_{1}=T g=d_{3} d_{4}$, with $g=(n n-1 n-2 n-3 n-4)$ and

$$
\begin{aligned}
& d_{3}=\left(a_{1} \cdots a_{n-5} n-2 n n-3 n-1 n-4\right), \\
& d_{4}=\left(b_{1} \cdots b_{n-5} n n-3 n-1 n-4 n-2\right) .
\end{aligned}
$$

Note that $d_{3}$ and $d_{4}$ are in the same class, be it $C$ or $C^{\prime}$, in $A_{n}$. By again using $d_{1}^{\prime}$ and $d_{2}^{\prime}$ in place of $d_{1}$ and $d_{2}$, the proof is completed in this case.

In Case 2, $S$ has a conjugate $S_{1}$ such that $T=S_{1} g^{-1}$ fixes at least 5 letters. Hence without loss of generality the factors $d_{1}, d_{2}\left[d_{1}^{\prime}, d_{2}^{\prime}\right]$ can be chosen so that $T=d_{1} d_{2}=d_{1}^{\prime} d_{2}^{\prime}$ with

$$
\begin{array}{ll}
d_{1}=\left(a_{1} \cdots a_{n-6} n-5 n-4\right), & d_{1}^{\prime}=\left(a_{1}^{\prime} \cdots a_{n-6}^{\prime} n-5 n-4\right) \\
d_{2}=\left(b_{1} \cdots b_{n-6} n-4 n-5\right), & d_{2}^{\prime}=\left(b_{1}^{\prime} \cdots b_{n-6}^{\prime} n-4 n-5\right)
\end{array}
$$

and where $a_{i} \rightarrow b_{i}\left[a_{i}^{\prime} \rightarrow b_{i}^{\prime}\right]$ is an odd permutation of the letters $1,2, \cdots, n-6$. Now $S_{1}=T g=d_{3} d_{4}$, where

$$
\begin{aligned}
& d_{3}=\left(a_{1} \cdots a_{n-6} n-1 n-5 n-3 n-2 n n-4\right), \\
& d_{4}=\left(b_{1} \cdots b_{n-6} n-5 n-2 n n-3 n-4 n-1\right) .
\end{aligned}
$$

The permutations $d_{3}$ and $d_{4}$ belong to the same class in $A_{n}$. Priming the $a_{i}$ and $b_{i}$ completes the proof in this case. 
In Case 3, $S$ has at least two 3-cycles, and has a conjugate $S_{1}$ such that $T=S_{1} g^{-1}$ fixes the letters $n, n-1, n-2, n-3, n-4, n-5$. By the induction hypothesis permutations $d_{1}$ and $d_{2}$ exist such that $T=d_{1} d_{2}$ with

$$
\begin{aligned}
& d_{1}=\left(n-4 a_{1} \cdots a_{k} n-5 a_{k+1} \cdots a_{n-6}\right), \\
& d_{2}=\left(n-4 b_{1} \cdots b_{l} n-5 b_{l+1} \cdots b_{n-6}\right),
\end{aligned}
$$

and where $d_{1}$ and $d_{2}$ are in the same class in $A_{n}$. (We cannot assume that $n-4$ and $n-5$, which are fixed by $T$, are neighbors in $d_{1}$ and $d_{2}$, but it is possible that $k=0$ and $l=n-6$ or that $k=n-6$ and $l=0$.) Now $S_{1}=T g=d_{3} d_{4}$, where

$$
d_{3}=d_{1} h, \quad d_{4}=h^{-1} d_{2} g
$$

with $h=(n-5 n-3 n-2)(n-4 n-1 n)$. Then $d_{3}$ and $d_{4}$ are both $n$-cycles. It has only to be checked that they are in the same class in $A_{n}$; to do this is tedious, but straightforward. To complete the proof in this case we observe that since $S$ contains two 3-cycles and $S_{1}=d_{3} d_{4}$, the decomposition $S_{1}=d_{3}^{\prime} d_{4}^{\prime}$ can be obtained by applying a certain outer automorphism of $A_{n}$.

In the only remaining case, $S$ fixes 2 letters, and therefore has a conjugate $S_{1}$ such that $T=S_{1} g^{-1}$ fixes

$$
n, n-1, n-2, n-3, n-4 \text {. }
$$

Again we have $T=d_{1} d_{2}$, where we can write

$$
d_{1}=\left(a_{1} \cdots a_{n-6} n-4 n-5\right), \quad d_{2}=\left(b_{1} \cdots b_{n-6} n-5 n-4\right),
$$

and where the permutation $a_{i} \rightarrow b_{i}$ is an odd permutation of the letters $1,2, \cdots, n-6$. Then $S_{1}=T g=d_{3} d_{4}$, with

$$
\begin{aligned}
& d_{3}=\left(a_{1} \cdots a_{n-6} n-1 n n-3 n-2 n-4 n-5\right), \\
& d_{4}=\left(b_{1} \cdots b_{n-6} n-5 n-4 n n-2 n-3 n-1\right),
\end{aligned}
$$

and these belong to the same class. By priming we again conclude $C C$ contains $S$, and the proof is complete in all cases. Hence Theorem 1.

5. Covering $A_{16 k}$. By means of an almost identical argument we have shown that the class $C$ of type $4 l_{1} \quad 4 l_{2} \quad 4 l_{3} \quad 4 l_{4}\left(l_{i} \geqq 1\right)$ in $A_{n}\left(n=4 \Sigma l_{i}\right)$ has the covering property (1). The lemma required is simpler: Let $m=4 l, b=(12 \cdots m)$. Taking $x=$ 


$$
(m m-3 m-2 m-1, m-4 m-7 m-6 m-5, \cdots, 8567,4123)
$$
gives

$$
b x=(13)(2 m)(46)(57) \cdots(m-4 m-2)(m-3 m-1) .
$$

Hence if $D$ is the class of type $4 l_{1} 4 l_{2} \cdots 4 l_{r}$ ( $r$ even) in $A_{n}$, then $D D$ contains the type $2^{n / 2}$.

In order to start the induction we had to prove that the class $C$ of type $4^{4}$ has the property $C C=A_{16}$. The calculations are too lengthy to be included. (A copy can be had from any of the authors.) This yields Theorem 2 .

One can ask how small a period is possible for a class $C$ with property (1). The first result in this direction was that of $X u$ [4] who found such a class with period $n-3$ if $n$ is odd and period $n-2$ if $n$ is even. From the result of Bertram quoted in the introduction, it follows that the smallest period of such $C$ is $\leqq 3 n / 4$. While Theorem 2 does not give covering for all $n$, it nevertheless yields, among classes $C$ in $A_{n}$ satisfying (1),

$$
\liminf _{n \rightarrow \infty} \frac{\text { period of } C}{n} \leqq \frac{1}{4}
$$

as opposed to Bertram's $3 / 4$.

From the other direction we have shown [3] that for $n>6$ there is no class $C$ in $A_{n}$ having property (1) and period 2, and if $n=12 k+10$ there is no such class of period 3. There may be such a class of period 4 , however. More precisely, we conjecture that for $n=8 k$, the class $C=4^{2 k}$ has the covering property (1).

\section{REFERENCES}

1. E. A. Bertram, Even permutations as a product of two conjugate cycles, J. Combinatorial Theory (A) 12 (1972), 368-380.

2. J. L. Brenner, Covering theorems for nonabelian simple groups. II, J. Combinatorial Theory (A), 14 (1973), 264-269.

3. J. L. Brenner, M. Randall, J. Riddell, Covering theorems for finite nonabelian simple groups. I, Colloq. Math. XXXII.1, 1974 (to appear).

4. Cheng-Hao Xu, The commutators of the alternating group, Sci. Sinica 14 (1965), 339-342.

Received August 20, 1973 and in revised form May 21, 1974. The first author was supported by NSF grant GP-32527. The third author was supported in part by NRC A-5208.

10 Phillips Road, Palo alto, Ca Arizona State University, Tempe AND

UNIVERSITY OF VICTORIA 


\section{CONTENTS}

Zvi Artstein and John A. Burns, Integration of compact set-valued functions

J. A. Beachy and W. D. Blair, Rings whose faithful left ideals are cofaithful

Mark Benard, Characters and Schur indices of the unitary reflection group $[321]^{3}$

H. L. Bentley and B. J. Taylor, Wallman rings ............................. 15

E. Berman, Matrix rings over polynomial identity rings II ............... 37

Simeon M. Berman, A new characterization of characteristic functions of absolutely continuous distributions ........................ 323

Monte B. Boisen, Jr. and Philip B. Sheldon, Pre-Prüfer rings ............ 331

A. K. Boyle and K. R. Goodearl, Rings over which certain modules are injective

J. L. Brenner, R. M. Crabwell and J. Riddell, Covering theorems for finite nonabelian simple groups. $V$

H. H. Brungs, Three questions on duo rings .................................... 345

Iracema M. Bund, Birnbaum-Orlicz spaces of functions on groups ....351

John D. Elwin and Donald R. Short, Branched immersions between 2-manifolds of higher topological type

J. K. Finch, The single valued extension property on a Banach space ................................................................................. 61

J. R. Fisher, A Goldie theorem for differentiably prime rings ............ 71

Eric M. Friedlander, Extension functions for rank 2, torsion free abelian groups ...................................................................... 371

J. Froemke and R. Quackenbusch, The spectrum of an equational class of groupoids

B. J. Gardner, Radicals of supplementary semilattice sums of

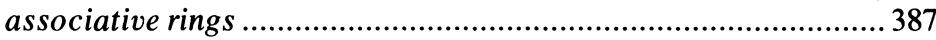

Shmuel Glasner, Relatively invariant measures ...............................393

G. R. Gordh, Jr. and Sibe Mardešić, Characterizing local connectedness in inverse limits...

S. Graf, On the existence of strong liftings in second countable topological spaces

S. Gudder and D. Strawther, Orthogonally additive and orthogonally increasing functions on vector spaces ........................................427

F. Hansen, On one-sided prime ideals .......................................... 79

D. J. Hartfiel and C. J. Maxson, A characterization of the maximal monoids and maximal groups in $\beta x$.

Robert E. Hartwig and S. Brent Morris, The universal flip matrix and the generalized faro-shuffle 


\section{Pacific Journal of Mathematics}

Vol. 58, No. 1

March, 1975

John Allen Beachy and William David Blair, Rings whose faithful left ideals are cofaithful .................................... 1

Herschel Lamar Bentley and Barbara June Taylor, Wallman rings ........ 15

Elizabeth Berman, Matrix rings over polynomial identity rings. II ...... 37

Ann K. Boyle and Kenneth R. Goodearl, Rings over which certain modules are injective ................................. 43

J. L. Brenner, Robert Myrl Cranwell and James Riddell, Covering theorems for finite nonabelian simple groups. $V \ldots \ldots \ldots \ldots \ldots \ldots \ldots \ldots \ldots$

James Kenneth Finch, The single valued extension property on a Banach

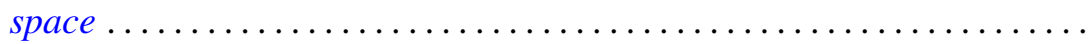

John Robert Fisher, A Goldie theorem for differentiably prime rings........ Friedhelm Hansen, On one-sided prime ideals .................... Jon Craig Helton, Product integrals and the solution of integral equations..........................................

Barry E. Johnson and James Patrick Williams, The range of a normal

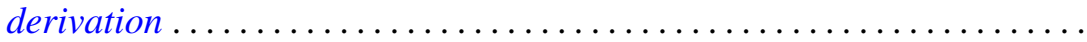

Kurt Kreith, A dynamical criterion for conjugate points ............ 123

Robert Allen McCoy, Baire spaces and hyperspaces .............. 133

John McDonald, Isometries of the disk algebra ................ 143

H. Minc, Doubly stochastic matrices with minimal permanents ......... 155

Shahbaz Noorvash, Covering the vertices of a graph by vertex-disjoint paths. ...

Theodore Windle Palmer, Jordan *-homomorphisms between reduced Banach*-algebras

Donald Steven Passman, On the semisimplicity of group rings of some

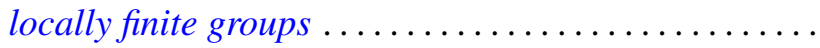

Mario Petrich, Varieties of orthodox bands of groups .

Robert Horace Redfield, The generalized interval topology on distributive

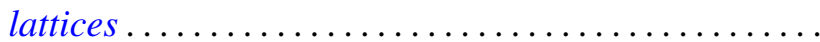

James Wilson Stepp, Algebraic maximal semilattices .... . .

Patrick Noble Stewart, A sheaf theoretic representation of rings with Boolean orthogonalities ........................

Ting-On To and Kai Wing Yip, A generalized Jensen's inequality......... 255

Arnold Lewis Villone, Second order differential operators with self-adjoint

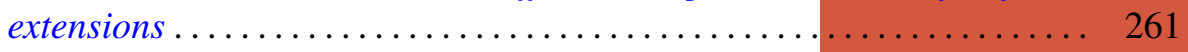

Martin E. Walter, On the structure of the Fourier-Stieltjes algebra ....... 267

John Wermer, Subharmonicity and hulls .................... 283

Edythe Parker Woodruff, A map of $E^{3}$ onto $E^{3}$ taking no disk onto a

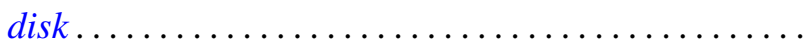

